Boyd, J. S. K. \& Bidwell, D. E. (1957). J. gen. Microbiol. 16, 217-228

\title{
The Type A Phages of Salmonella typhimurium: Identification by a Standardized Cross- immunity Test
}

\author{
By J. S. K. BOYD AND D. E. BIDWELL \\ The Wellcome Laboratories of Tropical Medicine, London, N.W. 1
}

\begin{abstract}
SUMMARY: The identification of the type A phages of Salmonella typhimurium has hitherto been based on antigenic structure, host range, and plaque characters. The discovery of an apparently phage-free strain of $S$. typhimurium, equally sensitive to all these phages, has enabled us to prepare standardized lysogenic bacteria, alike in all respects except in the symbiotic phage (prophage) they carry. With these it has been possible to test cross-immunity with a high degree of accuracy. The immunity pattern of a collection of 598 type A phages has been examined, and subdivisions in the original types have been revealed. Twelve types are now identified. The test is described and the significance of the findings is discussed.
\end{abstract}

In early papers (Boyd, 1950, 1954) some details were given of a group of heatresistant bacteriophages, derived from lysogenic strains of Salmonella typhimurium, which were designated type $\mathbf{A}$. These were found to be of three antigenic types, of which the first was common, the second less so, and the third rare. The first of these antigenic types was further divided in accordance with the affinity of its members for certain bacterial hosts; some of the phages were able to multiply on the two indicator strains then in use, S. typhimurium 1404 and $S$. typhimurium 1411, while the others multiplied only on 1411 . Subsequent work has shown that the types characterized in this way are not all homogeneous and that they can be further broken down by cross-immunity tests. This more extensive subdivision has added precision to the method of distinguishing types of $S$. typhimurium by identifying the symbiotic phage they carry (Boyd, Parker \& Mair, 1951). In the present paper a description is given of the development and technique of the cross-immunity test and of the results of its application in the classification of these phages.

\section{METHODS}

The culture media and methods of manipulation were thosedescribed in previous papers (Boyd, 1950, 1954). The importance of testing samples of different batches of the nutrient agar to be used, and, when a good sample is found, the convenience of securing a large supply sufficient for some months' work, is again emphasized. Because the phages under investigation are very heatresistant-they survive a temperature of $80^{\circ}$ for $30 \mathrm{~min}$.- it has been possible to dispense with filtration and to destroy bacteria and any co-existent $\mathbf{B}$ phages in phage preparations by heating to $70^{\circ}$ for $30 \mathrm{~min}$.

Lysogenic bacteria and type $A$ phages. The 598 type A phages used in this investigation were recovered from 1400 cultures of Salmonella typhimurium. 
The majority of these, when received in this laboratory, were recently isolated from infected material-chiefly from the faeces of cases of human infection, but a few from contaminated foods and other sources. 1020 cultures were sent to us by Dr M. T. Parker, and they comprise the majority of the strains isolated in the Manchester area between January 1950 and December 1954. Most of the remainder were from hospital and public health laboratories in different parts of the country, a very few being from overseas.

Indicator strain. An innovation of great importance was the use, in the later stages of the investigation, of the recently acquired indicator strain, Salmonella typhimurium Q1 (Boyd, 1956). As far as can be ascertained, Q1 contains no symbiotic phage: it is sensitive to all the known phages of $S$.typhimurium, of both $\mathbf{A}$ and $\mathbf{B}$ types. The $\mathbf{A}$ phages all multiply well on it and, under suitable conditions, produce a lysate containing a high concentration of free particles. Further, when the appropriate technique is used, Q1 can be 'symbiotically' infected with all these phages, and so rendered lysogenic. In this way a series of lysogenic cultures has been prepared, in all of which the bacterium is the same, and only the symbiotic phage (prophage) which they carry is different.

Preparation of phage suspensions and lysogenic strains of Q1. Phage suspensions and lysogenic strains of Q1 are prepared as follows:

(1) Inoculate the lysogenic Salmonella typhimurium (usually a subculture of the original strain recovered from infected material) into broth, and incubate overnight.

(2) Spin until clear: the supernatant fluid contains the free phage particles liberated from the lysogenic bacteria.

(3) Heat to $70^{\circ}$ for $30 \mathrm{~min}$. to kill any remaining bacteria and destroy $B$ phages (if present).

(4) Add $0.5 \mathrm{ml}$. of this supernatant fluid to $4.5 \mathrm{ml}$. of an actively growing broth culture of $Q 1$ containing approximately $10^{8}$ bacteria $/ \mathrm{ml}$. Incubate until clearing occurs, or overnight if there is no clearing.

(5) Spin and heat to $70^{\circ}$ for $30 \mathrm{~min}$.

(6) Titrate by making decimal dilutions in broth to $10^{-6}$. This can be done with sufficient accuracy by a dropping technique, using standardized pipettes. Flood a plate of nutrient agar with a well-grown culture of Q1, pipette off the excess, and allow the plate to dry. Place a loopful of each dilution of phage on a marked spot on the plate. Allow to dry. Incubate overnight.

(7) Examine with a plate microscope to ensure that only one type of A phage is present. In the very exceptional cases where two are present, one is usually less numerous than the other, and its scanty plaques can be seen superimposed on the coalesced plaques of the more common variety.

(8) Read the results of the titration. The plate will show all gradations from solid-centre 'patches' in the low dilutions to discrete plaques in the high. The Critical Test Concentration (CTC) is the highest dilution at which the plaques are completely confluent, so that the patch appears quite homogeneous and has a smooth edge. This point is likely to lie somewhere between two of the original decimal dilutions. If necessary, a further test with intermediate dilutions can be put up, but with experience there is little difficulty in 
estimating the correct point by gauging the density of the patch which just fails to show CTC.

(9) For test purposes, dilute the phage preparation to CTC.

(10) With a sterile platinum needle take a small inoculum of bacteria from the centre of the patch given by the $10^{-1}$ dilution. Suspend this in about $1 \mathrm{ml}$. of broth, and immediately plate from this on to nutrient agar. Incubate.

(11) Next morning the plate will show some colonies with markings which indicate phage contamination, and others which appear normal. Select one of the latter-they are almost without exception lysogenic-and inoculate into broth. Incubate.

(12) When this culture shows slight opalescence, sub-inoculate into a growing broth culture of Q1. After 3-4 hr. incubation, place a drop of this second culture on nutrient agar sown with $\mathrm{Q} 1$, and incubate overnight. The development of plaques or a patch will confirm that the culture is lysogenic.

(13) Plate from the broth culture made in (11), and incubate. A uniform crop of colonies of normal appearance, completely devoid of phage markings, confirms that it is uncontaminated by extraneous free phage.

(14) Make stock cultures from the colonies on this plate.

The points of fundamental importance in this somewhat elaborate technique are $(a)$ the phage particles from the original lysogenic strain at no time pass through any other host organism except $Q 1$, and $(b)$ the lysogenic strains of Q1 are identical except for the prophage they carry.

\section{RESULTS}

\section{Preliminary observations on cross-immunity}

We were first made aware of the existence of more than one type of Al phage by the chance observation that a freshly isolated temperate phage, which had been provisionally identified as $A 1$, produced typical patches and plaques when 'spotted' on a plate sown with a lysogenic strain carrying Al. As a lysogenic bacterium is 'immune' to the phage it produces, this new phage was clearly of a different type from that carried by the organism on which it was 'spotted'. Following this observation, a series of cross-immunity tests was carried out with all the phages in our collection which had been identified as A1. The results showed that they were of at least three different types. However, when lysogenic bacteria carrying these three types were exposed to the action of the other $\mathbf{A}$ phages, the results were not uniform, and the position was somewhat confused. The lysogenic bacteria used in these tests were subcultures of those isolated from faeces or other infected material.

Similar tests were then carried out on a cross-section of the many strains belonging to the type originally designated A2. (At this time the types now placed as A 2e and A2f had been identified but were classified separately.) The strains in this first sampling fell into three clear-cut types, but a few strains were found, when the complete collection was tested, which did not exactly match any of these types, and certain anomalous results, comparable to those seen in the Al series, were also encountered. 
Among the different factors capable of causing the minor irregularities in the reactions of the $\mathbf{A} 1$ and to a lesser extent the A2 types, it seemed possible that the host bacterium might play a part. Thus, there might be some difference in the receptor mechanism which is involved in the adsorption of the phage particles, or the bacterium might carry another not very obvious prophage which had nevertheless antagonistic or immunizing properties. Some support is given to this theory by the differing sensitivity of 1404 and 1411 to these A phages, neither of these indicators being capable of supporting all of them equally, though they all will grow to a greater or lesser extent on 1411. It is of interest to record that nothing has been discovered to explain the differing sensitivity of 1404 and 1411 to the A phages. Whether or not they carry a weak B prophage - this must be regarded as a possibility - neither carries an A prophage which could confer immunity. No difference has been found in their biochemical reactions, and Dr Joan Taylor reports that they are of identical antigenic structure. With the discovery of $Q 1$, which is equally sensitive to all the A phages, it became possible to by-pass the problem of variations attributable to the host bacterium. Using standardized lysogenic strains of Q1 prepared in the way that has been described and standardized suspensions of the temperate phages, the irregularities in the cross-immunity test disappeared.

The extent to which the different phages act on sensitive lysogenic bacteria is variable. Some appear much more active than others, and produce patches and plaques which are not to be distinguished, either in appearance or in number, from those they produce on phage-free Q1. Others produce shallow, weak plaques which are most easily seen by transmitted light, and which are present in greatly reduced numbers in comparison with the yield given on the indicator strain. Between these extremes are intermediate forms of varying activity.

Some of the strong lysates containing a high concentration of temperate phage particles exercise a lytic action on certain of the lysogenic bacterial strains. The lytic agent concerned is non-particulate, and its action fades out as the phage preparation is diluted, in most cases having disappeared when a dilution of $10^{-2}$ is reached. When present, the lysis tends to obscure the action of the weaker phages, and it has therefore been found advantageous to dilute phages which are to be tested for cross-immunity beyond the point where lytic action occurs. This is achieved, and at the same time the necessary degree of standardization is introduced, by diluting the phage to the Critical Test Concentration (CTC) of Craigie \& Yen (1938), as already described.

\section{Technique of the cross-immunity test}

The technique of the cross-immunity test is simple. A loopful of a broth culture of each of the lysogenic strains is spread over an area about an inch in diameter on the surface of nutrient agar in a Petri dish and allowed to dry, the back of the plate being marked so that the different cultures can be identified. On the centre of each inoculated area there is placed a standard loopful of the phage which is being tested, diluted to CTC; this in turn is allowed to dry, the plate incubated overnight, and the results read next day. The reaction may 
range from a solid patch through decreasing numbers of separate plaques to a negative result. Symbols ranging from ++++ to + and - are used to score the results. While phages of the same type may show some variation in the intensity of their action, attributable possibly to the adjustment of CTC, the overall pattern of reactions is constant.

\section{The cross-immunity pattern}

The compiled results of the cross-immunity test are set out in Table 1, in which for convenient reference the antigenic relationships and host range of the different types are also shown.

Twelve types have been differentiated. The original A1 and A2 types are each subdivided into four. A5 (Boyd, 1954) is now shown as A2e, as it has the same antigen and host range as the other A2 types. A sixth variety, which had been recognized as a separate type, but has not so far been described, is included as A2f. All strains of A3 appear to be identical. The position of A4 will be given in detail later.

Of the 598 strains of phage examined, 592 fall into these 12 types. The remaining 6 give anomalous results.

Ten of the 12 types (Table 1) have a distinctive cross-immunity pattern by which they can be readily identified. While some variations in the degree of the positive reactions may occur, this pattern is constant. Two of the phages (A2f and A3) do not react with any of the lysogenic strains-or, to put it the other way round, all the lysogenic strains are immune to these two phages. Though they cannot be directly identified by cross-immunity, they are readily distinguished by antigenic pattern, host range, and plaque characters.

It will be noted that the test can be performed in two ways, either by testing the unknown phage against the known lysogenic bacteria-the method which has been described - or, vice versa, by rendering Q1 lysogenic with the unknown phage and testing its immunity to known phages. Either method is equally reliable, but the former is preferred because it is both simpler and quicker to carry out, a standardized phage being easier to prepare than a lysogenic strain of Q1.

\section{Plaque characters of different types}

The characters of the plaque may be of some assistance in confirming the identity of a phage but they are apt to show considerable variation and must be interpreted with caution. The quality of the nutrient agar used, differences in the thickness of the agar layer (even the relatively minor differences resulting from a slightly convex or concave bottom to the Petri dish), the concentration of bacteria in the indicator culture with which the plate is sown-these and other factors influence the development of the phage and make it difficult to define the normal plaque. The typical plaque formed by all A phages appears as a saucer-shaped depression in the sheet of bacterial growth formed by the indicator strain. A little opaque dome-shaped mound of bacteria, like a small colony, occupies the centre of the saucer. Immediately surrounding this there is a narrow rim which may be comparatively clear, and which merges into 


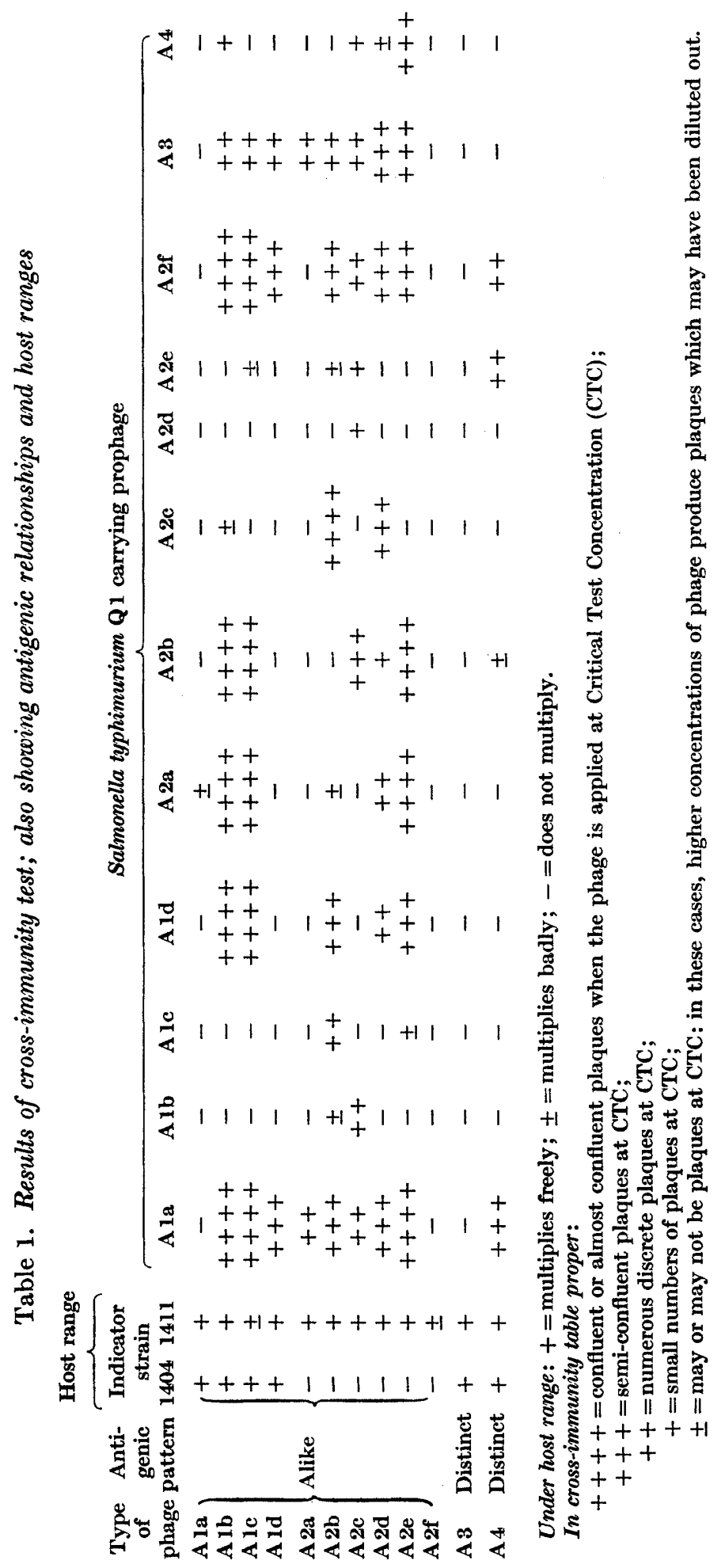


a zone of increasing opacity, the inner halo. The inner halo extends to the edge of the saucer and varies in width, depending on the steepness with which the saucer shelves. With certain phages, and certain indicator strains, there is a further partially clear zone-the outer halo-which surrounds the saucer and may be as wide as the plaque.

When Q1 is used as indicator, some of the types have characters which are sufficiently obvious and constant to be of value in confirming identity.

(1) The plaques of A2e and A2f are approximately half the size of the others, A2f being the smaller of the two. (On a good medium, the solid centres of the large-plaque types measure up to $1 \mathrm{~mm}$. across, while the outside measurements of the saucer are from 1.5 to $2 \mathrm{~mm}$.)

(2) A2b has a small rather irregular centre and a wide inner halo which may show concentric rings, giving it the appearance of a target.

(3) A4 has a characteristically granular rim and inner halo.

A2e, A3 and A4 have little or no outer halo, whereas in the other types this feature is well marked.

\section{Incidence of the different phage types}

Table 2 shows the numbers of the different phage types identified from 1948 to 1954, and Table 3 the localities in which the lysogenic strains of Salmonella typhimurium carrying these phages were isolated.

Table 2. Isolations of type A phages of Salmonella typhimurium by years

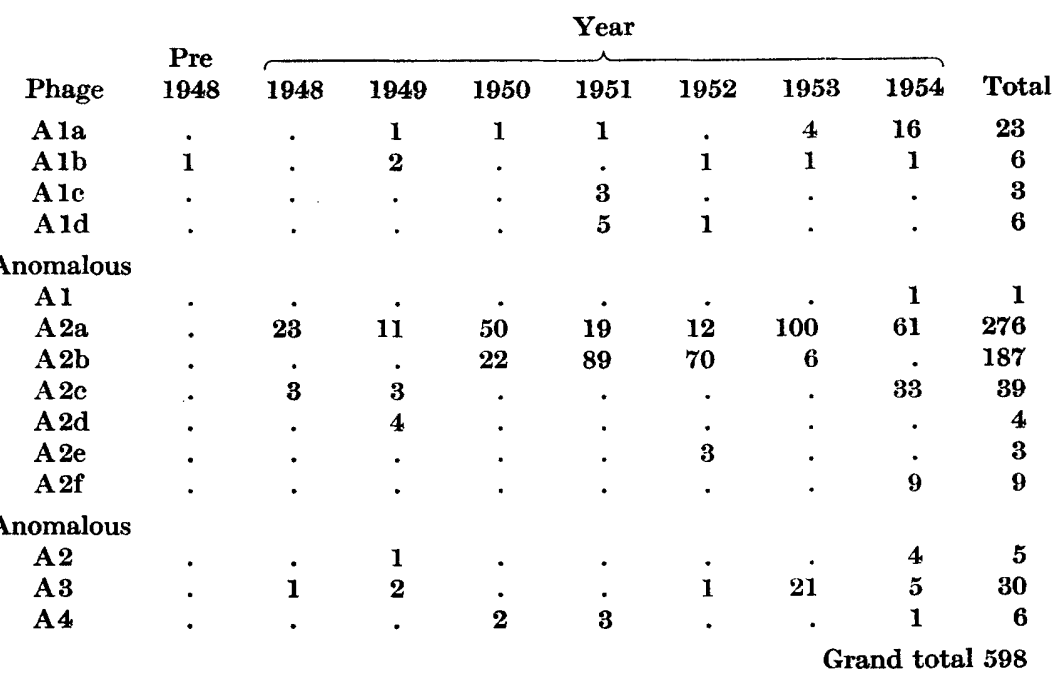

The figures indicate the number of strains of Salmonella typhimurium carrying each phage. With a few exceptions, these were isolated from infected material in the year indicated.

The original Ala, the first of these phages to be identified, was recovered from a specimen of faeces before the investigation recorded in this paper started, and was maintained as a lysogenized strain of 1404. It has since been 
recovered repeatedly from cultures of $S$. typhimurium, and is thus a wellestablished type, though not, in our experience, as common as certain of the A2 types.

Only a few Alb strains have been found, but they have cropped up in different years. One was kindly given to us by $\mathrm{Dr}$ B. A. D. Stocker. It is Lilleengen's LT22 strain 409 (Lilleengen, 1948), and was the strain used by Zinder \& Lederberg (1952) in their transduction experiments. It was originally isolated in Chile. In Lilleengen's experience this was a common type, providing 80 out of the 667 strains he examined, and being recovered from man, the horse and the hen.

Table 3. Geographical distribution of lysogenic Salmonella typhimurium strains carrying A phages

\begin{tabular}{ll} 
Phage & \multicolumn{1}{c}{ Localities in which the lysogenic bacteria have been isolated } \\
A1a & Portsmouth, Manchester \\
A1b & Chile, Liverpool, Manchester \\
A1c & Manchester \\
A1d & Manchester \\
A2a & Norwich, Copenhagen, Winchester, Stafford, Salisbury, Oxford, Liverpool, \\
& Portsmouth, Birmingham, Woolwich, Neasden, Ipswich, Manchester \\
A2b & Portsmouth, Liverpool, Manchester \\
A2c & Norwich, Liverpool, Manchester \\
A2d & Liverpool \\
A2e & Manchester \\
A2f & Manchester \\
A3 & Wakefield, Manchester \\
A4 & Germany, Manchester
\end{tabular}

Of the 1400 strains of Salmonella typhimurium examined, 1020 were from the Manchester area, and only $\mathbf{3 8 0}$ from elsewhere.

A 1c is closely related to Alb, and shows only minor differences in the crossimmunity pattern. The validity of these minor differences is confirmed by the plaque characters as revealed by transmitted light, for A 1c has a noticeably granular rim, which Alb lacks. Another difference is that Alc multiplies badly on the indicator strain, 1411. The three strains of A lc came from a common source and represent an isolated incident.

All six strains of Ald came from Manchester between August 1951 and February 1952. It seems probable that the infections from which they were derived were from a common source or were related one to the other.

A2a is the type first described as A2 (Boyd, 1950). It was found early in the investigation and has been one of the commonest types in the Manchester series, predominating in some years and relatively uncommon in others. It has also been found in strains of Salmonella typhimurium from other parts of the country, and is without doubt a stable and permanent type.

A 2b was not differentiated from A2a until the cross-immunity test was devised, although it had long been suspected that all the types designated A2 were not alike. Fluctuations in its incidence can be seen in Table 2. While the majority of the strains came from Manchester, several have been recovered 
from specimens from other parts of the country. As already mentioned, it has distinctive plaque characters.

A culture of Salmonella typhimurium carrying A2c was found in a batch of strains kindly sent by Lt.-Col. H. J. Bensted, Central Public Health Laboratory, Colindale, in 1948, and 2 strains came from Norwich in the same year. Three strains were received from Liverpool in 1949. This type did not, however, appear in the Manchester series until 1954, when 29 cultures of $S$. typhimurium carrying this phage were isolated.

The original number of A2d strains was 5: unfortunately 1 was lost before the cross-immunity test was brought into use. All 5 were from cultures from the same outbreak of enteritis, 4 from human infections and 1 from the rabbitpie which was responsible. When these phages were first examined, they were thought to be of the usual A2 types prevalent at the time, and no particular significance was attached to the incident. The cross-immunity test reveals an unusual source of infection which would not otherwise have been suspected.

The 3 strains of A2e were from the Manchester area and were encountered between July 1952 and February 1953. This is the only occasion on which this type has been found.

A2f was found in 9 strains of Salmonella typhimurium, all recovered in the Manchester area in fairly close sequence in October and November 1954. It may safely be assumed that they emanated from a common source.

A3, which has a distinctive antigen, has been found in cultures sent from different places at different times throughout the investigation. All strains have an identical cross-immunity pattern.

A 4 has also a distinctive antigen and has plaques of characteristic appearance. Nevertheless, cross-immunity tests divide this small group of 6 strains into 3 types. The first 2 (Boyd, 1950) come from bacteria isolated from human infections in Manchester. The cross-immunity results given in Table 1 are those of this pair. The second 3 strains were isolated from pigeons in Germany. These have a distinctive cross-immunity pattern. The last strain is of considerable interest. It occurs, together with an anomalous Al phage, in a strain of Salmonella typhimurium of human origin which is the only one in the series known to carry two type A phages. No difficulty has been experienced in isolating these phages in pure culture, and there is no valid reason to suggest that there has been any intermingling of the phage characters, though this naturally suggests itself as an explanation of the aberrations in crossimmunity.

Because of the very small numbers of A 4 isolated, they have not been shown as separate subtypes. This had best await further isolations.

Little need be said of the 6 phages which do not fit into this classification. One is the Al phage found in association with A4, which has just been mentioned. Of the 5 A2 group phages, 1 was received from the Central Public Health Laboratory, Colindale, and is of unknown origin. The other 4, of which 2 are alike, came from Manchester in 1954. All may well be isolated representatives of types which are common elsewhere. 


\section{DISCUSSION}

The results given by the standardized cross-immunity test are definite and consistent. The use of a common non-lysogenic 'host' on which temperate type A phages can be grafted to produce lysogenic strains, or, alternatively, can be propagated to produce high concentrations of free phage particles, has eliminated extraneous factors which might interfere with the accuracy of the test. The repeated isolations of phages which give identical reactions, in some cases at intervals of years, is good evidence both of the reliability of the test and of the validity of these types.

The frequency with which the different types have been recovered (i.e. the total number of strains of each type) is governed by epidemiological factors and does not necessarily have any other significance. Most of the types of which only small numbers have been found (e.g. Alc, A1d, A2d, A2e, A2f, A4) were recovered over a short period of time, each from a series of cases likely to have been related, whereas the more common types (A1a, A2a, A2b, A2c, A3 and, to a lesser extent, A 1b) have recurred in different places throughout the years.

As human infection with Salmonella typhimurium is usually food-borne, it is possible, if not indeed probable, that the rarer types have been introducedperhaps in foodstuffs such as dried eggs-in a strain of $S$. typhimurium from an area where this particular type is prevalent. If the resultant outbreak of enteritis in the community is effectually checked, and if none of those infected become carriers, both host-bacterium and phage will automatically disappear. On the other hand, the common and frequently recurring types are likely to be from strains of $S$. typhimurium which have become established in carriers in the areas which have been investigated (e.g. Manchester and district), where fresh outbreaks are caused from time to time by infection disseminated by these carriers. There is no evidence that the pathogenicity of $S$. typhimurium is in any way influenced by its phage content, and there can be little doubt that more extensive sampling over a wider area would reveal the locality in which those types, rare in our investigations, are of common occurrence either in man or in animals which can contaminate human food.

An alternative, but unlikely, explanation of our findings, is that the rare types result from some form of re-combination which takes place when one type of temperate phage multiplies in a bacterium carrying the prophage of another type. In vitro experiments, which will be recorded in greater detail in a later paper, have so far failed to reveal changes of type occurring in this way. In these negative experiments the numbers of bacteria and phage particles involved were massive: in natural surroundings, chance encounters between lysogenic bacteria and temperate particles to which they are sensitive must be, in comparison, of the greatest rarity. The possibility of rare types taking origin in this way cannot be ruled out, but so far has not been experimentally proved.

The repeated recovery of the more common types, particularly A1a, A2a, A2b and A3 in different parts of the country and at different times throughout the investigation, reveals the stability of these phage types in the lysogenic 
strains of Salmonella typhimurium which carry them. This is well illustrated in the case of A 2a and A2b. Both of these types commonly occur in association with B2, a coincidence which at first wrongly fostered the belief that all were alike and probably came from the same source. Differences in plaque characters and activity of growth on $\mathbf{1 4 1 1}$ were nevertheless observed, and it was at one time thought that some gradual change might be occurring in the character of the phage. This theory was decisively disproved by the cross-immunity test, which revealed two distinct varieties of A2 (A2c came into the picture at a later date), both of which had been present throughout the investigation, one predominating at one time and the other at another. As an interesting addendum nine strains of $S$. typhimurium from the Manchester area were sent for special investigation in 1955 : of these, eight were A2a and one was A2c. It is significant that these strains continue to maintain their identity, though they have been occurring in close association with each other in the same locality for a number of years.

Are further varieties of type A likely to be encountered? The answer to this query is definitely in the affirmative. The strains which have been noted as anomalous are, in all probability, types which are common in some other place, and there is no reason to doubt that there are others still to be found. This point can be settled only by widespread sampling and matching the reactions of the phages isolated against the standard pattern.

The wider range of phage types revealed by the cross-immunity test, and the certainty with which they can be identified, adds greatly to the reliability of the method of typing strains of Salmonella typhimurium described in a previous paper (Boyd et al. 1951). The value of the method will be further enhanced when the $B$ phages are also classified by a cross-immunity test of this kind. This test will not be as easy to carry out with $B$ phages as it has been with A phages, because the former are relatively heat-labile and therefore cannot be prepared in pure sterile culture by the simple process of heating. However, the difficulties are not insuperable, and it is hoped to undertake this work at some future date.

This method of phage-typing strains of Salmonella typhimurium, by isolating and identifying the symbiotic phage (prophage) they carry, is of greater accuracy than the modified Craigie method used by Lilleengen (1948) and by Felix (1956), which cannot properly cope with the subtleties introduced by multiple infections with A and B phages. Such multiple infections are of very common occurrence and result in many different and distinctive combinations, each of which breeds true. Nevertheless, the simple technique of the method used by Lilleengen and Felix and its accuracy in matching-as opposed to typing-lysogenic strains of $S$. typhimurium, make it a valuable test for routine investigations of a local nature. Its limitations were recognized by Felix himself, and led him to concur in the use of locally produced typing phages.

From the results shown in Table 1 it would appear that certain phages are much 'stronger' than others. Thus, strains of Salmonella typhimurium carrying A1b, A1c, A2d and A2e are resistant to the majority of the A phages, 
whereas the free phage which they produce can attack and multiply in many of the lysogenic bacteria of the group. Conversely, others-notably A Ia, A2f and A 3-confer little immunity on their host bacteria and have little capacity for attacking the other lysogenic bacteria. In another paper (Boyd, 1956) an outline has been given of the way in which these phages react with different lysogenic bacteria. In general, the 'strong' phages evict and replace the prophage of lysogenic bacteria carrying the weaker phages: the less strong phages may evict or may produce double infection, and, where this is reciprocal absence of immunity, double infection usually, but not invariably, results. These observations, details of which it is hoped to record in a later paper, have something in common with the findings of Bertani (1954), who worked with Shigella dysenteriae and mutants of a phage to which it is sensitive.

We are indebted to many bacteriologists throughout the country, and particularly to Dr M. T. Parker, for sending cultures of Salmonella typhimurium, to Dr Joan Taylor for a number of analyses of the antigenic structure of different strains, and to Mr R. A. Miles for much technical assistance in the earlier part of this investigation.

\section{REFERENCES}

Bertani, G. (1954). Studies on lysogenesis. III. Superinfection of lysogenic Shigella dysenteriae with temperate mutants of the carried phage. J. Bact. 67, 696.

Boyd, J. S. K. (1950). The symbiotic bacteriophages of Salmonella typhimurium. J. Path. Bact. 62, 501.

Boyd, J. S. K. (1954). The type A phages of Salmonella typhi-murium: two new types. J. Path. Bact. 68, 311.

BoYd, J. S. K. (1956). Immunity of lysogenic bacteria. Nature, Lond. 178, 141.

Boyd, J. S. K., Parker, M. T. \& MaIR, N. S. (1951). Symbiotic bacteriophage as a 'marker' in the identification of strains of Salmonella typhimurium. J. Hyg., Camb. 49, 442.

Craigie, J. \& Yen, C. H. (1938). The demonstration of types of B. typhosus by means of preparations of Type II Vi phage. Canad. publ. Hlth J. 29, 448, 484 .

Feuix, A. (1956). Phage typing of Salmonella typhimurium: its place in epidemiological and epizootiological investigations. J. gen. Microbiol. 14, 208.

Lilleengen, K. (1948). Typing of Salmonella typhi-murium by means of bacteriophage, Acta path. microbiol. scand. Suppl. 77.

Zinder, N. D. \& Lederberg, J. (1952). Genetic exchange in Salmonella. J. Bact. 64, 679 . 